\title{
Effects of Clearance around Square Pillar in Rectangular Enclosure on Cooling Performance of Pulsating Airflow
}

\author{
Takashi Fukue ${ }^{1}$, Koichi Hirose ${ }^{1}$, Hidemi Shirakawa ${ }^{2}$, Jun Suzuki ${ }^{3}$ and Yosuke Saga ${ }^{4}$ \\ 1. Department of Systems Innovation Engineering, Iwate University, Morioka, Iwate 020-8551, Japan \\ 2. Department of Mechanical Engineering, National Institute of Technology, Toyama College, Toyama 939-8630, Japan \\ 3. Department of Mechanical Engineering, Civil and Environmental Engineering, Graduate School of Iwate University, Morioka, \\ Iwate 020-8551, Japan \\ 4. Department of Die-Mold and Casting Engineering, Graduate School of Iwate University, Morioka, Iwate 020-8551, Japan
}

\begin{abstract}
This study focuses on a development of heat transfer enhancement techniques using pulsating flow for thermal equipment such as electronic equipment and heat exchangers. In this report, the heat transfer performance of the pulsating airflow around the heating pillar mounted in the rectangular enclosure was investigated experimentally while changing the size of the clearance between the enclosure wall and the pillar. The pillar simulates the components mounted in thermal equipment such as fins and electrical components. The rectangular enclosure simulates an enclosure of electronic equipment and heat exchangers. The shape of the cross section of the pillar was square having sides $30 \mathrm{~mm}$. The dimension of the width of the enclosure was changed from $50 \mathrm{~mm}$ to $80 \mathrm{~mm}$. It was found that the heat transfer performance of the pulsating airflow became higher than that of the steady flow regardless of the dimension of the clearance. The heat transfer enhancement around heating components by the pulsating flow can be available regardless of the clearance around the components.
\end{abstract}

Key words: Forced convection, heat transfer enhancement, pulsating flow, thermal management.

\section{Introduction}

Recent years have witnessed an increase in the demand for the reduction of power consumption in thermal equipment such as electronic equipment and heat exchangers in order to save energy. Generally, heat transfer performance of thermal equipment can be increased by supplying higher flow rate of working fluid. However, additional power consumption of fans and pumps is required to increase rotation speed and supply higher flow rate. In order to reduce power consumption of the fluid machineries while maintaining heat transfer performance, it is necessary to develop a novel control method of flow in thermal equipment.

Several studies of flow and heat transfer phenomena induced by pulsating flow [1-4] have shown the

Corresponding author: Takashi Fukue, research associate, doctor of engineering, research fields: heat transfer, fluids engineering, thermal design of electronic equipment. possibility of the heat transfer enhancement by the generation of flow pulsation. If we could generate a pulsating flow by controlling a rotation speed of fluid machineries, a heat transfer performance in thermal equipment would be improved while reducing time-averaged power consumption. The reduced net power consumption of the fluid machineries would lead to reductions of environmental road. Therefore, the generation of a pulsating flow by controlling the operation of the fluid machineries could be the next-generation technique for heat transfer enhancement in thermal equipment.

Our study tries to apply pulsating flow to thermal equipment in order to improve heat transfer performance while decreasing time-averaged power consumption of fans and pumps [5]. However, in order to apply the pulsating flow to thermal equipment, the effectiveness of the pulsating flow in thermal equipment should be evaluated. In densely packed 
electronic equipment, that are such as plasma displays [6], power supplies [7] and data centers [8], or compact heat exchangers [9], a number of components such as fins in the case of the heat exchangers are mounted in an enclosure and the structure of the flow passage become complex. Therefore, flow and heat transfer of a pulsating flow around the components mounted in the enclosure should be investigated in order to apply the pulsating flow to thermal equipment as a novel heat transfer enhancement technology.

We have been evaluating flow phenomena and heat transfer performance of pulsating airflow around the components mounted in the enclosure. In our previous reports $[5,10]$, we have reported that the pulsating airflow enhances heat transfer of the heating component mounted in the test enclosure while decreasing the time-averaged supply flow rate of the air regardless of the shapes of the component. In addition, we have confirmed the possibility of the generation of the pulsating flow by controlling the input voltage of the cooling fan periodically [11]. Through our previous reports, we confirmed the possibility of an application of the pulsating flow to thermal equipment as one of the heat transfer enhancement method.

Based on our previous investigation, in this report, we focused on an effect of a clearance between the component and the enclosure wall. The clearance is changed according to the dimensions of the component. This may affect flow pattern of the pulsating flow around the component. Especially, due to a miniaturization of thermal equipment such as portable electronic devices and compact heat exchangers, flow passages tend to become narrower. Therefore, we have to clarify whether the heat transfer enhancement by the pulsating flow is available regardless of the dimension of the flow passage or not.

Against these backgrounds, the heat transfer performance of the pulsating airflow around the heating pillar in the rectangular duct was investigated while changing the size of the clearance between the enclosure and the pillar. The pillar simulates a component mounted in thermal equipment and the duct simulates an enclosure of thermal equipment. Through the experiment, we obtained information about the relationship between the dimensions of thermal equipment and the effects of the pulsating flow on the heat transfer enhancement.

\section{Experimental Method}

\subsection{Wind Tunnel}

Fig. 1 shows the schematic of the wind tunnel that can generate pulsating airflow in a test section. The wind tunnel is composed of a pulsating flow generator, which consists of an $\mathrm{AC}$ servo motor and a cam, a flow-straightening chamber, an axial fan, a test section, which includes a test square pillar, and a flow mater. Cooling air is supplied by the axial fan that is mounted on the side wall of the chamber. The pulsating flow generator controls the opening area of the inlet of the test section by the rotation of the cam. By the change of the opening area of the inlet by the moving of the piston, the pressure drop at the inlet was changed, and the pulsating flow was generated. Pulsation frequency was controlled by the servo motor. Net supply flow rate was monitored with an ultrasonic flow meter (Aichi Tokei Denki, TRX25D-C/4P, range: $\pm 0.6 \sim 35 \mathrm{~m}^{3} / \mathrm{h}$, Accuracy: $\max \pm 5 \%$ R.D.).

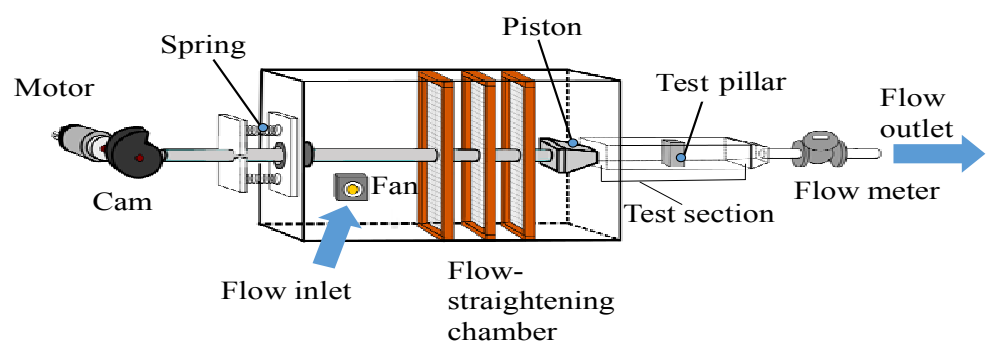

Fig. 1 Schematic of wind tunnel for measuring heat transfer of pulsating airflow. 


\subsection{Test Section}

Fig. 2 denotes the schematic of the test section. The dimensions of the test section simulate an enclosure of 1 U-size electronic equipment mounted on a 19-inch rack, which is the dimension standard of electronic equipment. The test section has a height of $40 \mathrm{~mm}$, a width of $80 \mathrm{~mm}$ and a length of $770 \mathrm{~mm}$. The square pillar, that was made of acrylic, was mounted on the test section. Acrylic plates were inserted symmetrically bilaterally in order to change the condition of the width of the test section. A stainless steel sheet heater was pasted around the obstruction in order to generate a constant heat flux. Three T-type thermocouples that have a diameter of $0.2 \mathrm{~mm}$ were installed between the obstruction surface and the stainless sheet heater. Positons of thermocouples were as shown in Fig. 3. Temperature data were collected every $0.1 \mathrm{~s}$ by using a data logger. By using the measurement results of temperature, the heat transfer performance around the obstruction was evaluated.

\subsection{Experimental Conditions}

Table 1 denotes the experimental conditions in this study. The width of the test section $b_{\mathrm{e}}$ was changed between $50 \mathrm{~mm}$ and $80 \mathrm{~mm}$. By changing the test section width, the effects of the clearance between the enclosure wall and the pillar on the heat transfer performance were investigated. Fig. 4 shows the time-dependent change of the supply flow rate when the pulsation frequency is $1 \mathrm{~Hz}$. The maximum supply flow rate when pulsating was set to $1.0 \times 10^{-3} \mathrm{~m}^{3} / \mathrm{s}$. Due to the closing the test section inlet, the time-averaged supply flow rate became $0.72 \times 10^{-3} \mathrm{~m}^{3} / \mathrm{s}$. The level of the supply flow rate is similar to a supply flow rate of 40 mm-scale small axial fans that are generally used in high-density packaging miniature electronic equipment. The pulsating frequency was set to $1 \mathrm{~Hz}$ and $2 \mathrm{~Hz}$. In order to evaluate the effectiveness of the flow pulsation, the heat transfer performance of the pulsating flow was compared with that of the steady flow that the time-averaged supply flow rate was the same as the pulsating flow.

\subsection{Evaluation Method of Heat Transfer Performance}

All heat transfer performance were evaluated by using time-averaged local Nusselt number at each temperature measurement point $N u_{\mathrm{n}}$ and time-averaged average Nusselt number for all temperature measurement point on the pillar $N u_{\mathrm{m}}$ as shown in the following formulas.

$$
N u_{\mathrm{n}}=\frac{\alpha_{\mathrm{n}} b_{\mathrm{o}}}{\lambda}[-]
$$

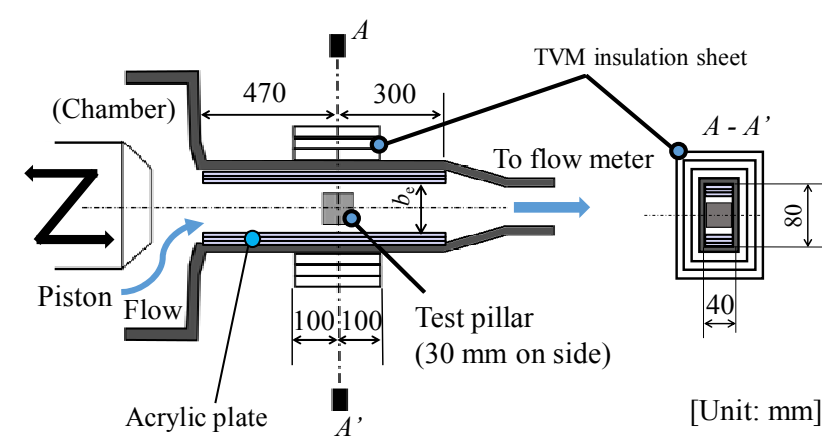

Fig. 2 Schematic of test section.

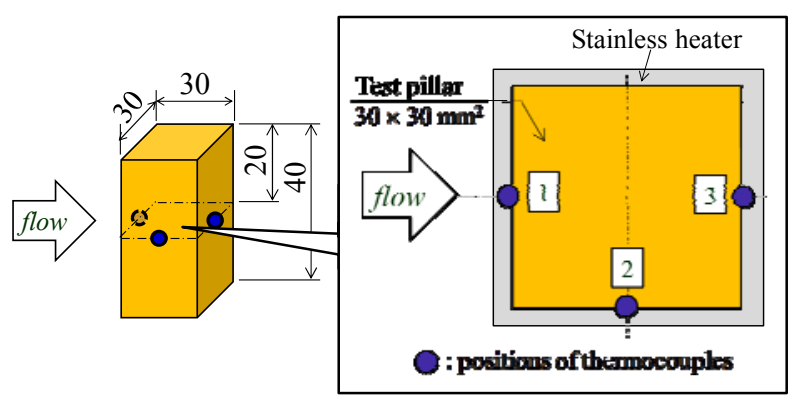

(a)

(b)

Fig. 3 Schematic of test pillar: (a) whole schematic and (b) cross section of test pillar and position of temperature measurement point by thermocouples.

\section{Table 1 Experimental conditions.}

\begin{tabular}{ll}
\hline Shape of obstruction & Square pillar \\
\hline $\begin{array}{l}\text { Width of test section } b_{\mathrm{e}}(\mathrm{mm}) \\
\text { Clearance between obstruction and }\end{array}$ & $50,60,70,80$ \\
$\begin{array}{l}\text { wall of test section }(\mathrm{mm}) \\
\text { Pulsation frequency }(\mathrm{Hz})\end{array}$ & $10,15,20,25$ \\
$\begin{array}{l}\text { Maximum supply flow rate when } \\
\text { pulsating }\left(\mathrm{m}^{3} / \mathrm{s}\right)\end{array}$ & $1.0 \times 10^{-3}$ \\
$\begin{array}{l}\text { Time-averaged supply flow rate when } \\
\text { pulsating }\left(\mathrm{m}^{3} / \mathrm{s}\right)\end{array}$ & $0.72 \times 10^{-3}$ \\
\hline
\end{tabular}




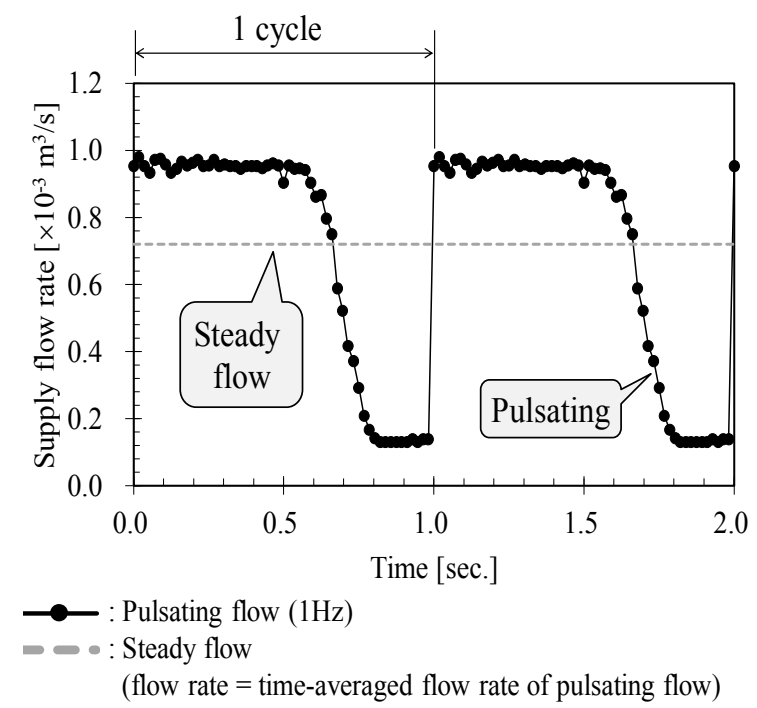

Fig. 4 Time-dependent change of supply flow rate in the case of pulsating flow.

$$
N u_{\mathrm{m}}=\frac{\alpha_{\mathrm{m}} b_{\mathrm{o}}}{\lambda} \quad[-]
$$

Here, $\mathrm{n}$ means the position of thermocouples, $\lambda[\mathrm{W} /(\mathrm{m} \cdot \mathrm{K})]$ is thermal conductivity of the air and $b_{\mathrm{o}}[\mathrm{m}]$ is the width of the test pillar. $\alpha_{\mathrm{n}}$ and $\alpha_{\mathrm{m}}$ are the local heat transfer coefficient and average heat transfer coefficient around the pillar respectively.

$$
\begin{aligned}
& \alpha_{\mathrm{n}}=\frac{Q_{\mathrm{n}}}{A_{\mathrm{n}} \Delta T_{\mathrm{n}}}\left[\mathrm{W} /\left(\mathrm{m}^{2} \cdot \mathrm{K}\right)\right] \\
& \alpha_{\mathrm{m}}=\frac{Q_{\mathrm{t}}}{A_{\mathrm{t}} \Delta T_{\mathrm{m}}}\left[\mathrm{W} /\left(\mathrm{m}^{2} \cdot \mathrm{K}\right)\right]
\end{aligned}
$$

where, $A_{\mathrm{n}}\left[\mathrm{m}^{2}\right]$ is a local heat transfer area on the pillar surface, that is the area of each face, $A_{\mathrm{t}}\left[\mathrm{m}^{2}\right]$ is a whole surface area of the pillar, $Q_{\mathrm{t}}[\mathrm{W}]$ is a total heat value from the pillar and $Q_{\mathrm{n}}[\mathrm{W}]$ is a local heat value from each face of the pillar $\left(Q_{\mathrm{n}}=Q_{\mathrm{t}} / 4\right)$. In this study, we assumed that the heat leakage by thermal conduction and radiation was enough smaller than the convection heat transfer on the pillar surface. Therefore, we assumed that all of the heat from the heater were dissipated by forced convection cooling on the pillar surface. $\Delta T_{\mathrm{n}}[\mathrm{K}]$ is a temperature difference between each measurement point of temperature on the pillar surface and the air at the test section inlet. $\Delta T_{\mathrm{m}}[\mathrm{K}]$ is a mean temperature difference of the all measurement points and the air at the test section inlet. Here, all temperature data were used as the time-averaged value.

The difference of heat transfer performance between the pulsating flow and the steady flow was evaluated by using the following performance ratio $\zeta_{\mathrm{m}}$.

$$
\zeta_{\mathrm{m}}=\frac{N u_{\mathrm{m}, \mathrm{p}}}{N u_{\mathrm{m}, \mathrm{s}}} \quad[-]
$$

Here, $N u_{\mathrm{m}, \mathrm{p}}$ is the average Nusselt number in the case of the pulsating flow and $N u_{\mathrm{m}, \mathrm{s}}$ is the average Nusselt number in the case of the steady airflow, respectively.

Here, we will confirm the validity of the use of the time-averaged values for evaluating the heat transfer performance of the pulsating flow by confirming the transient change of the local heat transfer coefficient when the temperature distribution reaches the steady state. Fig. 5 shows the transient change of local heat transfer coefficient between pulsating flow of $1 \mathrm{~Hz}$ and steady flow when $b_{\mathrm{e}}=80 \mathrm{~mm}$. Here, "Pulsating" denotes the transient change of the local heat transfer coefficient at each measurement point in the case of the pulsating flow of $1 \mathrm{~Hz}$ and "Steady" denotes the result of the steady flow (here, in this case, the supply flow rate of the steady flow was fixed on the maximum flow rate of the pulsating flow). We can see that the local heat transfer coefficient was not changed regardless of the existence of the pulsation. This is because the heat capacity of the test pillar is enough large and the instantaneous change of the heat transfer by the pulsating flow does not affect significantly to the transient temperature response on the pillar surface. Therefore, we concluded that we used time-averaged values as shown in Eqs. (2)-(6) for evaluating the heat transfer performance regardless of the existence of the pulsation. Here, the period of the measurement of the temperature was set to $2 \mathrm{~min}$ after achieving the steady state condition. 


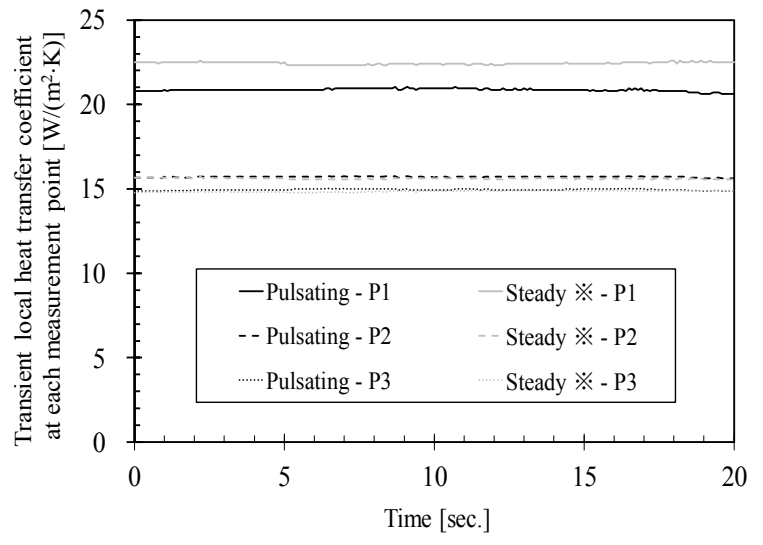

$※$ Supply flow rate was fixed on the maximum flow rate of the pulsating flow.

Fig. 5 Difference of transient change of local heat transfer coefficient between pulsating flow of $1 \mathrm{~Hz}$ and steady flow when $b_{\mathrm{e}}=80 \mathrm{~mm}$. Here, "P1" "P2" "P3" means the measurement point of temperature as shown in Fig. 3.

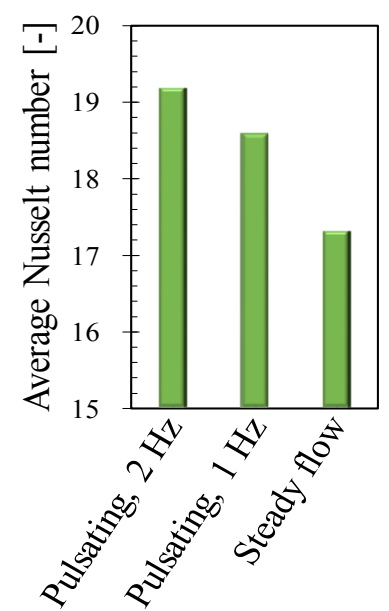

(a) Average Nusselt number

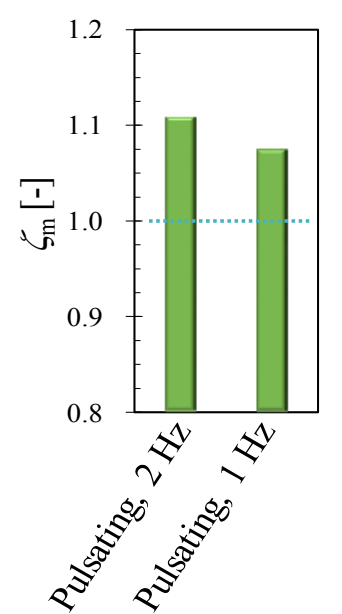

(b) Performance ratio
Fig. 6 Difference of average Nusselt number between pulsating flow and steady flow when $b_{\mathrm{e}}=80 \mathrm{~mm}$.

\section{Results and Discussion}

We will confirm the heat transfer enhancement by the pulsating flow. Fig. 6 shows the difference of average Nusselt number between the pulsating flow and the steady flow in the case of $b_{\mathrm{e}}=80 \mathrm{~mm}$. We can see that the average Nusselt number of the pulsating flow becomes about $10 \%$ higher than the result of the steady flow. The time-averaged supply flow rate of the steady flow was the same as the pulsating flow. However, the heat transfer performance became different. Therefore, we can say that the generation of the pulsation improves the heat transfer around the pillar. The reason of the heat transfer enhancement is as follows [5]. Generally, when the steady flow is supplied around the pillar, the flow separation causes on the side surface and the rear surface of the pillar. Then, the heat transfer performance decreases because of the airflow separation. However, due to the generation of the pulsation, the air flows to the side surface and the rear surface of the pillar as the static pressure in the flow separation area decreases due to the flow separation. This mechanism generates the counter flow and increases the heat transfer coefficient around the pillar. Therefore, the generation of the pulsating flow enhances the heat transfer behind the pillar where the flow separation generally occurs and heat transfer performance decreases.

Next, we will investigate the relationship between the width of the test section and the heat transfer coefficient. Fig. 7 shows the relationship between the width of the test section and whole heat transfer performance. We can confirm that a level of the heat transfer enhancement was dependent on the width. When the width became narrower, net heat transfer performance increased due to the acceleration of the airflow by the narrow clearance between the obstruction and the wall of the test section. However, from the viewpoint of the heat transfer enhancement effect by the pulsating flow, when the width of the test section became narrower, the performance ratio slightly decreased according to the test section width.

Fig. 8 shows the difference of the local heat transfer coefficient on each surface of the pillar. Here, "No." of the symbol means the position of the measurement point of thermocouples. Fig. $8 \mathrm{a}$ is the result in the case of $b_{\mathrm{e}}=80 \mathrm{~mm}$. On the front surface, the heat transfer on the front surface in the case of the pulsating flow decreased due to the decrease of the net supply flow rate in the case of the flow pulsation. However, due to the generation of the counter flow to the flow separation area, the heat transfer performance on the side surface and the rear surface improved and the net 


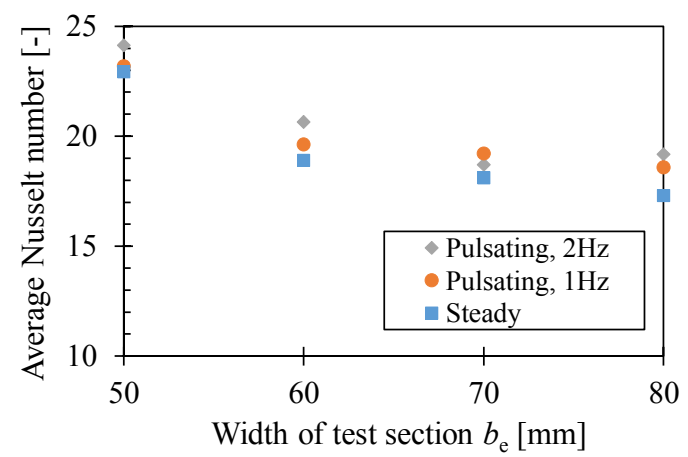

(a) Average Nusselt number

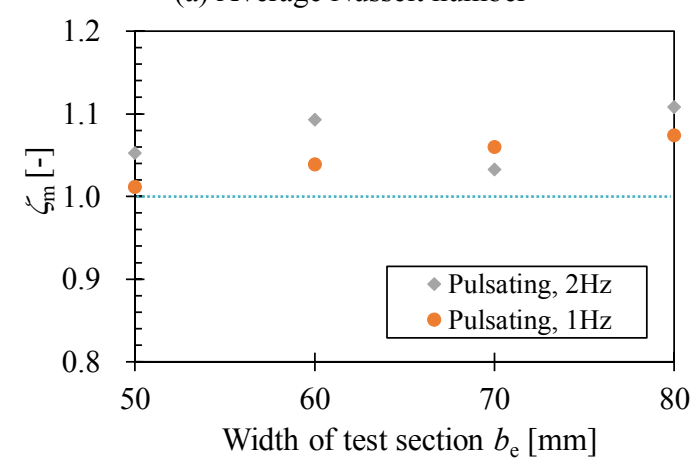

(b) Performance ratio

Fig. 7 Relationship between duct width and whole heat transfer performance.

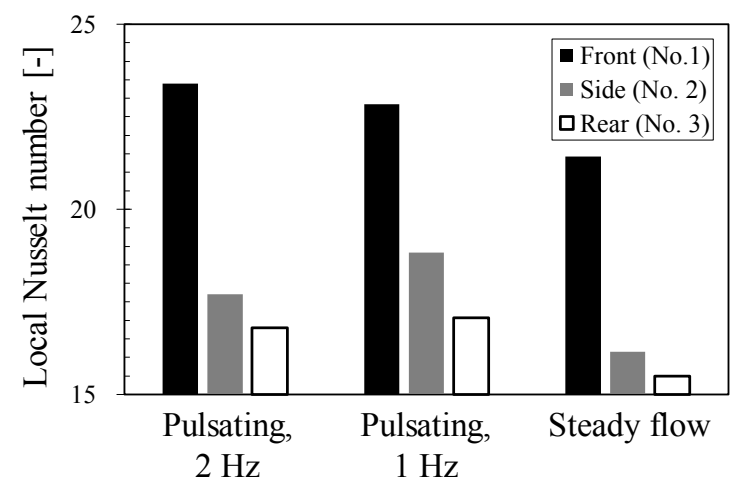

(a) $b_{\mathrm{e}}=80 \mathrm{~mm}$

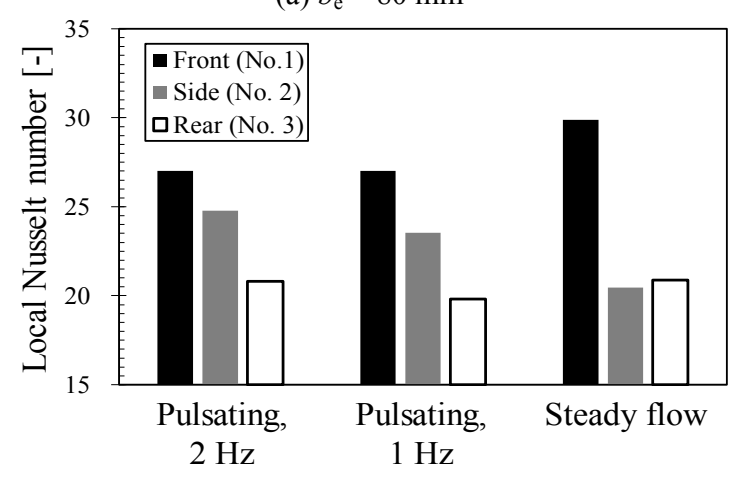

(b) $b_{\mathrm{e}}=50 \mathrm{~mm}$

Fig. 8 Change of local Nusselt number by flow pulsation. heat transfer of the pulsating flow increased. On the other hand, Fig. $8 \mathrm{~b}$ shows the result in the case of $b_{\mathrm{e}}=$ $50 \mathrm{~mm}$. In the case of the pulsating flow, the level of the heat transfer enhancement on the side surface increases but the heat transfer performance on the rear surface becomes smaller than the steady flow. By the narrow clearance between the obstruction and the wall, the generation of the flow separation area was inhibited and the enough counter flow did not generate. In addition, due to the acceleration of the main flow between the pillar and the wall, the airflow behind the pillar become complex regardless of the generation of the pulsating flow. Therefore, the level of the improvement of the heat transfer by the pulsating flow was inhibited.

\section{Conclusions}

In this study, we are investigating the flow and heat transfer of the pulsating airflow around the pillar mounted in the enclosure. In this paper, the relationship between the width of the test section and the level of the heat transfer enhancement was investigated. Especially, the heat transfer performance of the pulsating airflow around the pillar was evaluated experimentally while changing the width of the test section. Through the experiment, we obtained the undermentioned conclusions.

In the range of our research, heat transfer enhancement by the pulsating flow can be observed regardless of the width of the test section. A level of the heat transfer enhancement by the pulsating flow was dependent on the width of the test section. When the width of the test section became narrower, the level of the heat transfer enhancement slightly decreases. Especially, the flow condition on the side or rear surfaces of the pillar affects the net heat transfer performance of the pulsating flow around the pillar.

As our future research, the optimum condition of the pulsating flow in order to obtain maximum heat transfer enhancement effect should be evaluated. 


\section{Performance of Pulsating Airflow}

\section{Acknowledgment}

This work was supported by JSPS KAKENHI Grant Number JP16K18022.

The authors also wish to acknowledge the assistance of Ms. Natsuki Yatsu, the former student at Graduate School of Iwate University, Mr. Nobuaki Suzuki, the student at Graduate School of Iwate University, Ms. Wakana Hiratsuka and Mr. Taiki Furusawa, the students at Iwate University.

\section{References}

[1] Saitoh, H., and Yoshioka, Y. 2010. "Effect of Pulsating Amplitude on Flow Structure and Associated Heat Transfer around the Flat Plate Installed in Pulsating Duct Flow." Presented at the 21st International Symposium on Transport Phenomena, Taiwan.

[2] Kikuchi, Y., Ohno, Y., and Takahashi, M. 1995. "Combined Forced and Free Convective Heat Transfer from a Cylinder in Pulsating Cross-Flow." Transactions of JSME, Series B 61 (585): 202-7. (in Japanese)

[3] Sung, H. J., Hwang, K. S., and Hyun, J. M. 1994. "Experimental Study on Mass Transfer from a Circular Cylinder in Pulsating Flow." International Journal of Heat Transfer 37 (15): 2203-10.

[4] Inukai, A., Takahashi, M., Hishida, M., and Tanaka, G. 2005. "Characteristic of Heat Transportation by an Oscillatory Flow." Transactions of JSME, Series B 71 (710): 131-8. (in Japanese)

[5] Fukue, T., Hirose, K., and Yatsu, N. 2014. "Basic Study on Flow and Heat Transfer Performance of Pulsating Air Flow for Application to Electronics Cooling."
Transactions of the Japan Institute of Electronics Packaging 7 (1): 123-31.

[6] Isoshima, N., Watanabe, M., Ri, M., Yamada, Y., and Sugimoto, K. 2009. "Development of a Low-Noise and High-Performance Cooling Structure for Full-HD Plasma Display TV Sets." In Proceedings of the ASME InterPACK2009, Paper No. InterPACK2009-89041.

[7] Fukue, T., Hatakeyama, T., Ishizuka, M., Hirose, K., Obata, K., and Koizumi, K. 2015. "Flow Resistance Network Analysis in Fan-Cooled High-Density Packaging Electronic Equipment." In Proceedings of the ASME InterPACK/ICNMM2015, Paper No. InterPACK/ICNMM2015-48600.

[8] Billet, L. M., Healey, C. M., VanGilder, J. W., and Pardey, Z. M. 2015. "Data Center Cooling Efficiency with Simulation-Based Optimization." In Proceedings of the ASME InterPACK/ICNMM2015, Paper No. InterPACK/ICNMM2015-48425.

[9] Spitas, C., and Song, Y. 2010. "Thermal Analysis of the UCCHE-A Heat Exchanger using an Element Method with Localised Flow Properties." Presented at the 21st International Symposium on Transport Phenomena, Taiwan.

[10] Fukue, T., Hirose, K., and Shirakawa, H. 2015. "Basic Study on Cooling Performance of Pulsating Airflow around Components Mounted in High-Density Packaging Electronic Equipment (Effects of Shapes of Components on Cooling Performance)." In Proceedings of IEEE CPMT Symposium Japan (ICSJ), 48-51.

[11] Fukue, T., Hirose, K., Shirakawa, H., and Suzuki, J. 2015. "Possibility of Generation of Pulsating Flow and Heat Transfer Enhancement by Controlling Supply Voltage of Fans." In Proceedings of the JSME Thermal Engineering Conference 2016, Paper No. A134. (in Japanese) 